\title{
Indicadores do controle endócrino em vacas leiteiras de alta produção e sua relação com a composição do leite*
}

\author{
Indicators of endocrine control in high-yielding dairy cows and their relation with milk composition \\ Rómulo Campos', Félix Hilario Díaz González² ${ }^{2}$ Arlei Coldebella ${ }^{3}$ \& Luciana de Almeida Lacerda'
}

\begin{abstract}
RESUMO
A produção de leite é um desafio para o metabolismo energético. O controle endócrino regula a síntese e a secreção do leite, e a homeostasia da vaca leiteira. Os principais hormônios da lactação são cortisol, insulina, triiodotironina (T3), tiroxina (T4) e leptina. A mobilização de reservas lipídicas e gliconeogênicas garantem a síntese do leite. O objetivo do presente trabalho foi monitorar o controle endócrino pós-parto em vacas leiteiras de alta produção e avaliar a sua relação com a composição do leite. $\mathrm{O}$ estudo foi realizado em 110 vacas multíparas da raça Holandesa. O cálculo da dieta foi feito mediante software comercial, para conhecer o balanço energético em cada rebanho. O período pós-parto avaliado correspondeu às semanas: $2,5,8,11$. Em cada período foram coletadas amostras de sangue e de leite de sete animais em cinco rebanhos diferentes. Os hormônios foram dosados mediante radioimunoanálise de fase sólida. A composição do leite foi determinada mediante espectrofotometria de infra-vermelho próximo (NIRS). As análises estatísticas incluíram provas de correlação, análises de variância e comparação de médias. Não foi encontrada correlação entre os hormônios e os sólidos totais do leite.
\end{abstract}

Descritores: vacas leiteiras, endocrinologia, composição do leite, pós-parto.

\begin{abstract}
Milk production is a challenge for energy metabolism. Endocrine control regulates milk synthesis and secretion, and the homeostatic status in the dairy cow. The main hormones in lactation process includes cortisol, insulin, triodothyronine (T3), thyroxine (T4) and leptin. Mobilization of lipidic and gluconeogenic reserves are necessary for milk synthesis. The main objective of the present work was to monitor the endocrine postpartum control in high-yielding dairy cows and to evaluate the possible incidence of such control on milk composition. The study was done with 110 multiparous HolsteinFriesian dairy cows. The ration balance was done through a commercial software to know the individual herd energy status. The evaluated periods were 2, 5, 8 and 11 postpartum weeks. In each period blood and milk samples of seven cows per herd were collected. The hormones were determined by solid phase radioimmunoassay. Milk composition was analyzed by near infra-red spectrophotometry (NIRS). Statistical analysis included correlation test, analysis of variance and means comparison tests. No significant relationships were obtained among hormones and total solids in milk.
\end{abstract}

Key words: dairy cows, endocrinology, postpartum, milk composition. 


\section{INTRODUÇÃO}

Nos últimos anos aumentou a produção individual de leite por lactação [18]. Além do incremento da produção relatam-se transtornos metabólicos nas vacas leiteiras [13,21]. As situações que alteram a homeostasia ou a homeorrese podem afetar a produção de leite $[2,28,35]$. As vacas de alta produção podem ser definidas como sistemas biológicos caracterizados pela alta demanda de energia e a complexa regulação endócrina $[2,30,35]$. Diversos trabalhos têm demonstrado a participação da insulina na regulação energética de vacas leiteiras [28]. Recentemente foram postulados modelos de integração do balanço energético descrevendo o papel da insulina na sua regulação [30]. O cortisol tem sido investigado por seu papel gliconeogênico no peri-parto. Níveis séricos de cortisol têm sido relacionados com o metabolismo materno durante o pós-parto, fazendo parte de trabalhos sobre estresse e doenças metabólicas [13,31]. A leptina é possivelmente o hormônio de mais recente descobrimento e seu papel ainda não foi completamente elucidado. Participa no controle da mobilização das reservas adiposas e protéicas para compensar o balanço energético negativo (BEN) [15]. Em ruminantes, não existem evidências definitivas sobre a função da leptina [29]. Os hormônios triiodotironina (T3) e tiroxina (T4), têm sido indicados como responsáveis por processos endógenos de regulação energética, por sua ação sobre o consumo de oxigênio [1,32].

O objetivo do trabalho foi monitorar a dinâmica endócrina em vacas leiteiras de alta produção e determinar a possível influência hormonal sobre a composição do leite.

\section{MATERIAIS E MÉTODOS}

Neste trabalho foram selecionadas 140 vacas multíparas da raça Holandesa com produções superiores a $25 \mathrm{~kg} /$ dia pertencentes a rebanhos considerados como sistemas intensivos de produção para as condições do Sul do Brasil. Após exame clínico e de verificação das condições exigidas para o estudo, foram considerados 110 animais. A alimentação consistiu em silagem de milho e sorgo, forragem verde de Tifton (Cynodon nlemfuensis), concentrado e suplementação mineral. Para o cálculo do valor nutricional do alimento foram seguidas as recomendações do NRC (2001). A ração foi balanceada mediante o programa Spartan Ration Evaluator (Michigan State University).
O ecosistema de localização dos rebanhos correspondeu ao Planalto Médio do Estado do Rio Grande do Sul. Os animais provinham de núcleos comerciais com práticas controladas de alimentação e manejo em sistemas semiconfinados. Foram avaliados os períodos climáticos extremos para a zona temperada (inverno e verão). Os animais foram divididos em quatro grupos correspondentes às semanas $2,5,8$ e 11 de lactação. Em cada rebanho e para cada semana considerada, foram coletadas amostras de sangue e leite de sete animais. A coleta das amostras para todos os animais foi realizada no mesmo dia em cada rebanho.

Antes da coleta de sangue, foi realizado exame clínico para descartar animais com sinais de doença (problemas podais, gastrintestinais ou endometrite). Mediante análise dos registros foi verificado o período de pós-parto do animal e a produção de leite dos dias anteriores e a do dia da coleta. As amostras de sangue foram coletadas mediante venipunção coccígea em tubos com vácuo com heparina sódica, conservadas em refrigeração e centrifugadas (3000 rpm por 15 minutos) para a obtenção de plasma, o qual foi dividido em frações, identificado e congelado a $-20^{\circ} \mathrm{C}$ até as determinações hormonais. As amostras de leite foram coletadas mediante sistema acionado do tanque individual, na ordenha da tarde, em recipientes com preservante bronopol (2-bromo,2-nitro-1,3 propanediol) e enviadas antes de 24 horas para o Serviço de Análises de Rebanhos Leiteiros (SARLE) da Universidade de Passo Fundo, onde mediante citometria de fluxo foi determinada a contagem de células somáticas (CCS) e por espectrofotometria de infra-vermelho próximo (NIRS), foi determinada a composição de gordura, proteína, lactose e sólidos totais.

A dosagem de cortisol ${ }^{1}$, insulina ${ }^{1}, \mathrm{~T}^{1}, \mathrm{~T}^{1} \mathrm{e}$ leptina ${ }^{2}$ foi realizada usando kits comerciais mediante radioimunoanálise (RIA) de fase sólida. Foi usado um contador gama automático para a quantificação radiativa. Para o cálculo da concentração foi usado o programa RIACALC ${ }^{\circledR}$ (Universidade de Guelph, Ontario, Canada). A análise estatística incluiu análises de variância (GLM) para conhecer o efeito das variáveis semana do pós-parto e rebanho, e prova de t para amostras independentes para o efeito época Foi realizada prova de correlação de Pearson, para avaliar a associação entre os hormônios e entre os componentes do leite. Para as análises estatísticas se utilizou o programa SAS [34]. 


\section{RESULTADOS}

Na Tabela 1 são apresentadas as informações sobre a composição do leite nos núcleos de produção do estudo. Em nenhum caso houve efeito significativo do período pós-parto sobre as variáveis estudadas. A variável época (inverno, verão) apresentou efeito significativo sobre os parâmetros produção de leite, sólidos totais e sólidos não gordurosos no leite. Foi observado efeito significativo do rebanho sobre os sólidos totais, sólidos não gordurosos e proteína. Os sólidos totais do leite refletem os principais aspectos da síntese do leite. Por uma parte, a lactose é considerada o "marca-passo" da produção láctea [26]. Por outra parte, a proteína e a gordura são a base fundamental das características nutricionais da secreção e consti- tuem os determinantes nas políticas de preços do produto [16]. Pelos critérios expostos, adotou-se a avaliação dos sólidos totais como a base para estudar a possível relação entre as variáveis endócrinas e metabólicas analisadas no presente trabalho.

Na Tabela 2 apresentam-se os valores plasmáticos dos principais hormônios associados ao controle endócrino da lactação em vacas leiteiras de alta produção para cada uma das semanas do estudo.

Na Tabela 3 apresentam-se os valores de correlação entre os sólidos totais no leite e os hormônios associados ao metabolismo energético. As informações em conjunto permitem inferir que os indicadores endócrinos não apresentam correlação linear com a característica selecionada para avaliar a composição do leite. As relações básicas entre sólidos totais do lei-

\begin{tabular}{|c|c|c|c|c|c|}
\hline \multirow{2}{*}{ Componente } & \multicolumn{4}{|c|}{ Semana de lactação } & \multirow{2}{*}{$\mathbf{P}$} \\
\hline & 2 & 5 & 8 & 11 & \\
\hline Gordura (\%) & $3,62 \pm 0,10$ & $3,77 \pm 0,12$ & $3,59 \pm 0,09$ & $3,55 \pm 0,09$ & 0,4445 \\
\hline Proteína (\%) & $2,95 \pm 0,04$ & $2,84 \pm 0,04$ & $2,86 \pm 0,05$ & $2,91 \pm 0,04$ & 0,2892 \\
\hline Lactose (\%) & $4,56 \pm 0,03$ & $4,56 \pm 0,04$ & $4,58 \pm 0,05$ & $4,65 \pm 0,03$ & 0,2745 \\
\hline STOTAL $^{1}(\%)$ & $11,66 \pm 0,13$ & $11,57 \pm 0,14$ & $11,47 \pm 0,12$ & $11,58 \pm 0,12$ & 0,8049 \\
\hline $\mathrm{SNG}^{2}(\%)$ & $8,03 \pm 0,10$ & $7,79 \pm 0,11$ & $7,88 \pm 0,10$ & $8,03 \pm 0,11$ & 0,2940 \\
\hline $\mathrm{IPG}^{3}$ & $0,84 \pm 0,02$ & $0,78 \pm 0,03$ & $0,82 \pm 0,03$ & $0,80 \pm 0,02$ & 0,3492 \\
\hline $\mathrm{NUL}^{4}(\mathrm{mmol} / \mathrm{L})$ & $3,49 \pm 0,28$ & $3,74 \pm 0,30$ & $3,26 \pm 0,34$ & $3,42 \pm 0,39$ & 0,7923 \\
\hline Produção 5 (L/d) & $32,6 \pm 1,3$ & $34,8 \pm 2,11$ & $34,13 \pm 1,65$ & $29,85 \pm 1,58$ & 0,1808 \\
\hline $\operatorname{ccS}^{6}$ & $296 \pm 70,18$ & $521 \pm 139,7$ & $271 \pm 90,34$ & $411 \pm 114,0$ & 0,3223 \\
\hline
\end{tabular}

${ }^{1}$ STOTAL $=$ Sólidos totais, ${ }^{2} \mathrm{SNG}=$ Sólidos não gordurosos, ${ }^{3} \mathrm{IPG}=$ Indice Proteína:Gordura, ${ }^{4} \mathrm{NUL}=$ Uréia no leite, ${ }^{5}$ Produção $=$ média invididual diária, ${ }^{6} \mathrm{CCS}=$ Contagem de células somáticas em $10^{3}$ células $/ \mathrm{mL}$.

Tabela 2. Valores plasmáticos médios ajustados e erro padrão para os principais hormônios associados a homeostasia de vacas leiteiras de alta produção.

\begin{tabular}{cccccc}
\hline \multirow{2}{*}{$\begin{array}{c}\text { Semana da } \\
\text { lactação }\end{array}$} & \multicolumn{5}{c}{ Hormônios } \\
\cline { 2 - 6 } & Cortisol $(\mathbf{n m o l} / \mathbf{L})$ & T3 $(\mathbf{n m o l} / \mathbf{L})$ & T4 $(\mathbf{n m o l} / \mathbf{L})$ & Insulina $(\mathbf{p m o l} / \mathbf{L})$ & Leptina $(\mathbf{n g} / \mathbf{m L})$ \\
\hline $\mathbf{2}$ & $41,11^{\mathrm{a}} \pm 5,79$ & $1,78^{\mathrm{a}} \pm 0,06$ & $31,20^{\mathrm{a}} \pm 1,09$ & $2,52^{\mathrm{a}, \mathrm{b}} \pm 1,24$ & $4,37^{\mathrm{a}} \pm 0,16$ \\
$\mathbf{5}$ & $25,11^{\mathrm{a}, \mathrm{b}} \pm 3,59$ & $2,03^{\mathrm{a}, \mathrm{b}} \pm 0,10$ & $36,10^{\mathrm{a}, \mathrm{b}} \pm 1,79$ & $2,26^{\mathrm{a}} \pm 0,97$ & $4,41^{\mathrm{a}} \pm 0,24$ \\
$\mathbf{8}$ & $13,52^{\mathrm{b}, \mathrm{c}} \pm 3,31$ & $1,89^{\mathrm{a}, \mathrm{b}} \pm 0,12$ & $39,42^{\mathrm{b}} \pm 2,06$ & $2,29^{\mathrm{a}, \mathrm{b}} \pm 0,88$ & $4,09^{\mathrm{a}} \pm 0,22$ \\
$\mathbf{1 1}$ & $7,72^{\mathrm{c}} \pm 2,21$ & $2,22^{\mathrm{b}} \pm 0,10$ & $41,32^{\mathrm{b}} \pm 1,51$ & $2,87^{\mathrm{b}} \pm 1,36$ & $4,76^{\mathrm{a}} \pm 0,27$ \\
$\mathbf{P}$ & $<0,001$ & 0,0010 & $<0,001$ & 0,0263 & 0,2070 \\
\hline Médias seguidas por letras diferentes nas colunas diferem significativamente pelo teste de Tukey $(\mathrm{P}<0,05)$.
\end{tabular}


Tabela 3. Coeficientes de correlação entre a porcentagem de sólidos totais do leite e entre os hormônios reguladores da síntese láctea em vacas de alta produção.

\begin{tabular}{|c|c|c|c|c|c|c|}
\hline & Cortisol & T3 & T4 & Insulina & Leptina & Sólidos totais \\
\hline Cortisol & 1,00 & & & & & \\
\hline T3 & $-0,14211^{\text {ns }}$ & 1,00 & & & & \\
\hline T4 & $-0,17292^{\text {ns }}$ & $0,69966^{\star \star}$ & 1,00 & & & \\
\hline Insulina & $0,19419^{*}$ & $-0,05601^{\text {ns }}$ & $-0,08579^{\text {ns }}$ & 1,00 & & \\
\hline Leptina & $-0,26646^{*}$ & $0,14817^{\text {ns }}$ & $0,20525^{*}$ & $0,11928^{\text {ns }}$ & 1,00 & \\
\hline Sólidos totais & $-0,01357^{\text {ns }}$ & $0,04833^{\text {ns }}$ & $-0,03110^{\text {ns }}$ & $-0,09418^{\text {ns }}$ & $0,03206^{\mathrm{ns}}$ & 1,00 \\
\hline
\end{tabular}

te e hormônios constituem a base de modelos para encontrar um indicador endócrino confiável para predizer a composição do leite. Os indicadores exibiram correlação negativa ou próxima de zero.

\section{DISCUSSÃO}

Os valores encontrados dos principais componentes do leite correspondem ao informado pela literatura para a raça Holandesa sob condições controladas de produção [2,37]. Os resultados mostram que os rebanhos selecionados dentro do chamado "sistema especializado" de produção de leite, alcançam um produto similar e dentro dos padrões exigidos pela Instrução Normativa 51 [24] exceto para o indicador sólidos livres de gordura cuja exigência é de $8,4 \%$, valor que não foi atingido em nenhum período.

A composição do leite não variou significativamente, sendo uma das características exigidas no comércio internacional de lácteos [16]. Diversos fatores têm sido associados a mudanças na composição láctea $[20,26]$. Entretanto, nos últimos anos, quando devido à seleção genética a produção individual atingiu $9000 \mathrm{~kg} / \mathrm{lactação,} \mathrm{possíveis} \mathrm{variações} \mathrm{individuais}$ ligadas a mudanças metabólicas têm sido pesquisadas [29]. Um dos fatores responsáveis tanto pela produção de leite, como pela homeostasia do animal tem sido o aporte de energia na ração $[4,26]$. Da mesma forma, os desequilíbrios energéticos são responsáveis, em grande medida, pelas chamadas "doenças da produção", as quais afetam a qualidade do leite $[9,13]$.

Diferentes autores têm investigado a composição do leite em relação a indicadores metabólicos $[1,25,29]$. Em geral, todos concordam em que a relação direta entre os indicadores de nutrição, os parâmetros metabólicos, o controle endócrino e a composição do leite não são fáceis de associar. Na literatura existem exemplos de esforços em modelar a síntese e a secreção do leite $[23,35]$. Nesses modelos se apresentam associações matemáticas que nem sempre conseguem ser explicadas com sentido biológico. Talvez essa seja a mais árdua tarefa dos futuros modelos nos quais, sob uma óptica mecanicista, a interação biológica entre metabólitos e componentes da nutrição possa explicar variações na composição do leite. A dinâmica própria das células da glândula mamária para síntese e secreção dos componentes do leite impedem uma relação única e linear entre cada metabólito e a soma dos sólidos, pois cada componente segue um padrão particular. Por esta razão, se encontram modelos únicos que estudam separadamente a composição de gordura, proteína ou lactose [21].

O controle da síntese, secreção e ejeção do leite reúnem um grande número de hormônios, entre os quais estão insulina, cortisol, tiroxina, triiodotironina e leptina $[28,29,36]$. O cortisol tem sido considerado como um bom indicador de estresse e, embora não seja o indicador ideal, constitui um valioso recurso no estudo das alterações endócrinas do pós-parto em função de seu papel estimulador da gliconeogênese e manutenção da glicemia [6]. Os valores de cortisol encontrados (Tabela 2) dão informação sobre os períodos de maior risco metabólico. A segunda semana de lactação apresentou os maiores valores de cortisol plasmático, enquanto que a semana 11 apresentou o menor valor. Esse comportamento é esperado e explica algumas patologias como a cetose e a laminite que têm relação direta com períodos de altos níveis de cortisol [11]. Um estudo ao redor do parto mostrou que os maiores valores de cortisol ocorreram no dia do parto e que muitas vacas mantiveram altos níveis ainda na terceira semana pós-parto [27]. Alguns autores postulam a possível ação do CRH na 
depressão do consumo de alimento no pós-parto, o que poderia explicar em parte as variações do comportamento individual para enfrentar o balanço energético negativo (BEN) no início da lactação [17]. Se considera que o cortisol varia em diferentes circunstâncias, razão pela qual, poderia não ser a melhor forma de avaliar o estresse [33].

A triidotironina (T3) e a tiroxina (T4) têm sido monitoradas em diferentes trabalhos em vacas leiteiras $[2,28]$. Os hormônios tireoidianos fazem parte de mecanismos homeostáticos responsáveis pela regulação térmica e o consumo de oxigênio nos diferentes tecidos. No presente trabalho, foi encontrada uma alta correlação entre T3 e T4 ( $\mathrm{r}=0,7, \mathrm{P}<0,01)$, concordando com outros trabalhos $[2,27,28]$, o que permite sugerir que a análise de apenas um hormônio poderia ser usada. Entretanto, a prova de correlação mostrou que a T4 e a leptina tiveram uma maior relação positiva, pelo que seria mais útil a determinação de T4, embora seja conhecido que a T3 é o hormônio ativo nos tecidos [7]. T3 e T4 alcançaram o menor valor na segunda semana pós-parto, como foi observado por Reist et al. [29], quem atribuem o menor valor a um possível efeito do BEN. Nas semanas seguintes do pósparto os dois hormônios apresentaram resultados similares, sendo os maiores valores observados na semana 11 (Tabela 2). Os valores durante todo o período avaliado estiveram dentro de valores relatados por outros autores [29], porém foram inferiores aos de outros trabalhos feitos no Brasil [3]. Os resultados permitem sugerir que os processos metabólicos de alta exigência comprometem os níveis circulantes dos hormônios tireoidianos e que, na medida em que os processos fisiológicos são compensados e diminui a pressão metabólica para a síntese de leite, conseguem elevar sua concentração no sangue.

A insulina tem sido considerada como o principal hormônio regulador da glicemia em mamíferos, mesmo em ruminantes onde ocorre um fluxo constante de precursores gliconeogênicos a partir do rúmen $[14,19]$. O interesse pela insulina durante o pós-parto está relacionado com a adequada síntese de lactose. Foi discutido que existe uma hipertrofia de alguns tecidos durante a lactação, entre estes o fígado. Esta hipertrofia é explicada pela necessidade de maior síntese de glicose. Da mesma forma, foi encontrado no mesmo trabalho, um aumento nos receptores celulares para insulina [19]. Um interesse adicional é sua possível participação na síndrome de depressão de gordura no leite [4]. Na década de 1990 foi postula- do que a insulina poderia estimular a síntese de tecido adiposo em órgãos diferentes à glândula mamária, diminuindo os precursores para a síntese de triglicerídeos no leite. Porém, outros trabalhos não confirmaram esta hipótese [5,12]. No presente trabalho, não foi encontrada relação da insulina com os sólidos totais do leite nem com a gordura do leite. Similares resultados são apresentados por outros autores em condições de balanço energético controlado $[1,12,14,27$, 29]. Na semana 11 ocorreu um aumento significativo dos níveis circulantes de insulina, possivelmente associado à redução da produção e à estabilização do consumo de alimento, como foi observado em outros trabalhos [2,17]. Houve uma relação positiva entre o cortisol e a insulina $(r=0,194 ; \mathrm{P}<0,05)$, o que tem explicação fisiológica na medida em que o cortisol promove a gliconeogênese. Valores altos de cortisol foram observados na segunda semana de lactação, período correspondente à fase ascendente da produção de leite. A insulina esteve positivamente relacionada ao BEN concordando com outras observações $[1,29]$.

A leptina é atualmente o centro dos estudos em endocrinologia de animais de produção [22]. Nos últimos anos, graças ao desenvolvimento de radioimunoanálise específico para ruminantes, ocorreram avanços no conhecimento deste hormônio [8]. Os primeiros trabalhos associaram seus efeitos ao desempenho reprodutivo e a lipomobilização [15]. Recentemente a pesquisa sobre leptina em gado de leite se concentrou na sua relação com BEN, variação na condição corporal e composição do leite [10,30]. Alguns trabalhos informam valores de leptina durante a lactação [36]. O presente trabalho é o primeiro no Brasil a apresentar valores de leptina durante a lactação de vacas de alta produção (Tabela 2), sendo similares a outros trabalhos [36]. O teor de leptina parece não ter grandes variações em função de dietas com níveis diferentes de energia [29]. Postula-se que os efeitos da leptina seriam através do sistema nervoso central bloqueando a ação do neuropeptídeo $\mathrm{Y}$, razão pela qual participaria mais na regulação homeorrésica que na homeostática, não apresentando sensíveis variações circulantes [22]. Apesar da teoria que a insulina aumentaria a expressão da leptina, no presente trabalho não houve relação entre esses dois hormônios. Porém, houve relação entre cortisol e leptina $(r=0,266 ; \mathrm{P}<0,01)$ e entre leptina e T4 $(r=0,205 ; \mathrm{P}<0,05)$. Esta última relação poderia entender-se pela presença de substratos energéticos de alta capacidade oxidativa, mobilizados e utilizados em resposta ao BEN. 
Não houve efeito significativo do rebanho sobre as variáveis endócrinas, exceto para a insulina $(\mathrm{P}<0,001)$. A maioria dos trabalhos menciona variações hormonais devidas ao balanço energético e por efeito de mastite [27,28]. Os rebanhos do presente estudo foram selecionados por suas condições padronizadas de produção, sanidade e manejo, sendo esta a possível causa da falta de efeito do rebanho sobre os hormônios analisados.

\section{CONCLUSÕES}

A relação estudada entre os principais hormônios da lactação e os sólidos totais do leite não permi- te inferir que exista um único hormônio relacionado com os parâmetros analisados. Foram determinadas importantes correlações de alguns hormônios entre si. Os resultados sugerem que os hormônios tireoidianos e a leptina são parte ativa da regulação energética da lactação. Os valores de leptina constituem os primeiros valores de referência para bovinos leiteiros no Brasil.

\section{NOTAS INFORMATIVAS}

${ }^{1}$ ICN Biomedicals; Irving, EUA.

${ }^{2}$ Linco Research, St Charles, EUA.

\section{REFERÊNCIAS}

1 Aeberhard K., Bruckmaier R.M. \& Blum J. 2001. Metabolic, enzymatic and endocrine status in high-yielding dairy cows. Part 2. Journal Veterinary Medicine Series A. 48: 111-127.

2 Aeberhard K., Bruckmaier R.M., Kuepfer U. \& Blum J. 2001. Milk yield composition, nutrition, body conformation traits, body condition scores, fertility and diseases in high-yielding dairy cows. Part 1. Journal Veterinary Medicine Series A.48: $97-110$.

3 Arcaro Jr. I., Arcaro J.R.P., Pozzi C.R., Fagundes H., Matarazzo S.V. \& Oliveira C.A. 2003. Teores plasmáticos de hormônios, produção e composição do leite em sala de espera climatizada. Revista Brasileira de Engenharia Agrícola e Ambiental. 7: 350-354.

4 Bauman D.E. \& Griinari J.M. 2003. Nutritional regulation of milk fat synthesis. Annual Review of Nutrition. 23: 203-227.

5 Baumgard L.H. 2003. Managed Milk Fat Depression. Tucson: University of Arizona, pp.41-45.

6 Beerda B., Kornalijnslijper J.E., Van der Werf J.T.N., Noordhuizen-Stassen E.N. \& Hopster H. 2004. Effects of milk production capacity and metabolic status on HPA function in early postpartum dairy cows. Journal of Dairy Science. 87: 2094-2102.

7 Cunningham J.G. 1997. Tratado de Fisiologia Veterinária. Capítulo V. Endocrinologia. Rio de Janeiro: Guanabara Koogan, pp.273-305.

8 Delavaud C., Bocquier F., Chilliard Y., Keisler D.H., Gertler A. \& Kann G. 2000. Plasma leptin determination in ruminants: effect of nutritional status and body fatness on plasma leptin concentration assessed by a specific RIA in sheep. Journal of Endocrinology. 165: 519-526.

9 Enjalbert F., Nicot M.C., Bayourthe C. \& Moncoulon R. 2001. Ketone bodies in milk and blood of dairy cows: Relationship between concentrations and utilization for detection of subclinical ketosis. Journal of Dairy Science. 84:583-589.

10 Feuermann Y., Mabjeesh S.J. \& Shamay A. 2004. Leptin affects prolactin action on milk protein and fat synthesis in the bovine mammary gland. Journal of Dairy Science. 87: 2941-2946.

11 Goff J.P. \& Horst R.L. 1998. Physiological changes at parturition and their relationship to metabolic disorders. Journal of Dairy Science. 80: 260-1268.

12 Griinari J.M., McGuire M.A., Dwyer D.A., Bauman D.E. \& Palmquist D.L. 1997. Role of insulin in the regulation of milk fat synthesis in dairy cows. Journal of Dairy Science. 80: 1076-1084.

13 Herdt T.H. 2000. Variability characteristics and test selection in herd level nutritional and metabolic profile testing. Veterinary Clinics of North America: Food Animal Practice.16: 387-403.

14 Holtenius K., Agenas S., Delavaud C. \& Chilliard Y. 2003. Effect of feeding intensity during the dray period. 2. Metabolic and hormonal responses. Journal of Dairy Science. 86: 883-891

15 Houseknecht K.L., Baile C.A., Matteri R.L. \& Spurlock M.E. 1998. The biology of leptin: A review. Journal of Animal Science. 76: 1405-1420.

16 Ibarra A. 2004. Sistema de pagamento do leite por qualidade: visão global. In: Dürr J.W., Carvalho M. \& Santos M.V. (Eds). O Compromisso com a qualidade do leite no Brasil. Passo Fundo: Universidade de Passo Fundo, pp.72-86.

17 Ingvartsen K.L. \& Andersen J.B. 2000. Integration of metabolism and intake regulation: a review focusing on periparturient animals. Journal of Dairy Science. 83: 1573-1597. 
18 Ingvartsen K.L., Dewhurst R.J. \& Friggens N.C. 2003. On the relationship between lactational performance and health: is it yield or metabolic imbalances that cause production disease in dairy cattle: A position paper. Livestock Production Science. 83: 227-308.

19 Kelly J.M., Summers M., Park H.S., Milligan L.P. \& McBride B.W. 1991.Celular energy metabolism and regulation. Journal of Dairy Science. 74: 678-694

20 Kennelly J.J., Glimm D.R. \& Ozimek L. 2000. Milk Composition in the Cow. Alberta: University of Alberta, 20p.

21 Kida K. 2003. Relationships of metabolic profiles to milk production and feeding in dairy cows. Journal of Veterinary Medicine Science. 65: 671-677.

22 Macajavá M., Lamosova D. \& Zeman M. 2004. Role of leptin in farm animals: a review. Journal Veterinary Medicine Series A. 51: 157-166.

23 McNamara J.P. \& Baldwin R.L. 2000. Estimation of parameters describing lipids metabolism in lactation: Change of existing knowledge described in model of metabolism. Journal of Dairy Science. 83: 128-143.

24 Ministério de Agricultura, Pecuária e Abastecimento. 2002. Brasil. Instrução Normativa No. 51. Brasília.

25 Mottram T. 1997. Automatic monitoring of the health and metabolic status of dairy cows. Livestock Production Science. 48: 209-217.

26 Mühlbach PR. 2000. Aspectos nutricionais que interferem na qualidade do leite. In: Prates E.R., Mühlbach P.R., Ospina H. \& Barcellos J.O. (Eds). Novos Desafios para a Produção Leiterira do Rio Grande do Sul. Porto Alegre: Universidade Federal do Rio Grande do Sul, pp.73-102

27 Nikolic J.A., Kulcsar M., Kátai L., Nedic O., Jánosi S. \& Huszenicza G. 2003. Periparturient endocrine and metabolic changes in healthy cows and in cows affected by mastitis. Journal Veterinary. Medicine Series A. 50: 22-29

28 Reist M., Erdin D.K., VonEuw D., Tschümperlin K.M., Leuenberger H., Chilliard Y., Hammon H.M., Morel C., Philipona C., Zbinden Y., Kuenzi N. \& Blum J. 2002. Estimation of energy balance at the individual and herd level using blood and milk traits in high-yielding dairy cows. Journal of Dairy Science. 85: 3314-3327.

29 Reist M., Erdin D.K., VonEuw D., Tschümperlin K.M., Leuenberger H., Delavaud C., Chilliard Y., Hammon H.M., Kuenzi N. \& Blum J. 2003. Concentrate feeding strategy in lactating dairy cows: metabolic and endocrine changes with emphasis on leptin. Journal of Dairy Science. 86: 1690-1706.

30 Reist M., Erdin D.K., VonEuw D., Tschümperlin K.M., Leuenberger H., Hammon H.M., Morel C., Philipona C., Zbinden Y., Künzi N. \& Blum J. 2003. Postpartum reproductive function: association with energy, metabolic and endocrine status in high yielding dairy cows. Theriogenology. 59:1-1707-1723.

31 Rivier C. \& Rivest S. 1991. Review. Effect of stress on the activity of the hypothalamic-pituitary-gonadal axis: peripheral and central mechanisms. Journal of Dairy Science. 45: 523-532.

32 Roche J.R., Kolver E.S. \& Kay J.K. 2005. Influence of precalving feed allowance on periparturient on metabolic and hormonal responses and milk production in grazing dairy cows. Journal of Dairy Science. 88: 677-689.

33 Rushen J. 2000. Some issues in the interpretation of behavioral responses to stress. In: Moberg G.P. \& Mench J.A. (Eds). The Biology of Animal Stress. Wallinford: CAB International, pp.23-42.

34 SAS Institute. 2001. SAS User's Guide: Statistics. Cary: SAS Institute Inc., 285p.

35 Sauvant D. 1994. Modelling homeostatic and homeorhetic regulations in lactating animals. Livestock Production Science. 39: $105-113$.

36 Soliman M., Ishioka K., Yoshida R., Komabayashi K., Hatai H., Matsui Y., Hirai T., Katagiri S., Takahashi Y., Kawakita Y.,Abe H., Kitamura H. \& Saito M. 2002. Serum leptin levels during the periparturient period in cows. Journal of Veterinary Medicine Science. 64: 1053-1056.

37 Wastra P. \& Jenness R. 1986. Química y Física Lactológica. Zaragoza: Acribia, 487p.

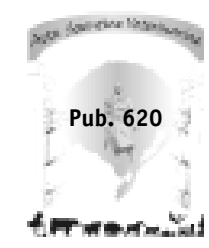

